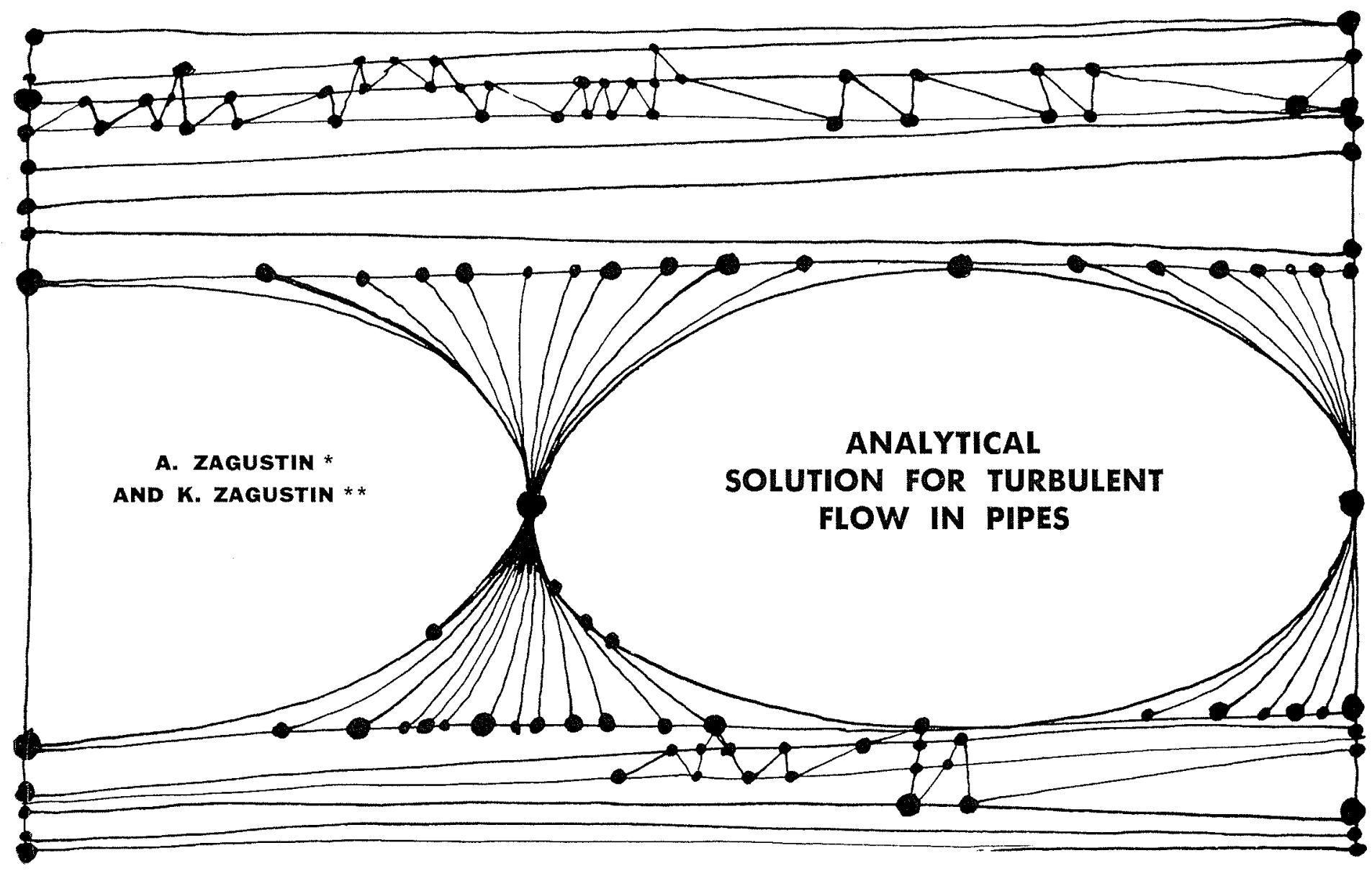

Introduction

There have been many papers presented on turbulent flow, but in most of them an empirical assumption on the mixing length distribution is introduced. The best known universal distribution laws for turbulent flow in pipes are those of Prandtl and Von Karman. Prandtl deduced the velocity distribution law from his mixing length theory. He assumed a proportionality between the mixing length and wall distance at the neighbourhood of the wall. This assumplion is reasonable, but Prandtl introduced an additional, far reaching assumption, namely that the mixing length distribution remain linear up to the center line of the pipe. The resultant velocity distribution is in good agreement with experimental data in the region close to the wall, but has a singularity at the center line. Von Karman made an attempt to determine the dependance of the mixing length on the space co-ordinate and he made the assumption that the mixing length is a function of the velocity distribution only. As in Prandtl's expression the resulting equation produces an angle at the axis.

In the present paper a new "balance of pulsation energy" equation is used. It represents the balance between the rate of energy lost due to molecular friction and the flux of turbulent energy produced at the wall. This equation was introduced for the first time by Anatol Zagustin in his paper on tur-

\footnotetext{
* Titular Professor, Faculty of Engineering, Central University of Vemezuela.

* K.Ph.D., Agregate Professor, Head Hydraulic Laboratory, Central University of Venezuela.
}

bulence published in 1938 in Russia $\left(^{* * *}\right.$ ), and it completes the set of differential equations necessary for the analytical solution of the problem. A remarkable property of this solution is that it depends only on the Karman universal constant $x$. The resulting curves for mixing length, velocity and eddy viscosity distributions are in good agreement with experimental data at any point of the pipe, including the center line. In particular the velocity distribution curve has a zero tangent at the center line and for the central region of the pipe coincides with the Darcy's empirical equation.

Since the solution was oblained from a set of basic equations and gives such good agreement with experimental data obtained from pipes, it is possible that the same idea could be used for solving other problems of steady turbulent flow.

\section{General theory}

The flow considered is assumed to be incompressible and as usual in working with fully developed turbulent motion the values of velocity, pressure and other properties will be considered as timeaverage, so that:

and :

$$
\overrightarrow{\mathrm{V}}(\vec{r}, t)=\overline{\overrightarrow{\mathrm{V}}}+\overrightarrow{\mathrm{V}}^{\prime}(\vec{r}, t)
$$

$$
\overline{\overrightarrow{\mathrm{V}}}=\lim _{\mathrm{T} \rightarrow \infty} \frac{\int_{t}^{t+\mathrm{T}} \overrightarrow{\mathrm{V}} d t}{\mathrm{~T}}
$$

(**) A. Zagustix. - Equations for the turbulent motion. Trudy Voronezhkogo Gosudarstvenogo Universiteta, $t . \mathrm{X}$ (1938), p. 5-39. 
in which $\vec{V}$ is the vector velocity, $\vec{r}$ denotes the position vector, $t$ denotes the time, $\mathrm{T}$ is an interval of time, $\overline{\vec{V}}$ is the time-average value of the velocity and $\vec{V}^{\prime}$, the pulsation of the particle, has an average value equal to zero.

Since all the properties will be considered as timeaverage, the bar indicating the average values will be avoided in the present paper.

The basic equations for turbulent flow in polar coordinates are expressed as follows:

\section{Continuity equation.}

In the case of a circular pipe the equation of continuity is expressed as:

$$
\frac{d u}{d r}+\frac{u}{r}=0
$$

in which $u$ is the $r$-component of the velocity vector.

\section{Navier-Stokes equation.}

Time-average notation is introduced and Boussinesq's concept of the "apparent" or "eddy" viscosity $A_{\tau}$ is used to form:

$$
\tau_{l}=\mathrm{A}_{\tau} \frac{d u}{d r}
$$

where $\tau_{t}$ denotes the turbulent shearing stress. This expression is similar to:

$$
\tau_{l}=\mu \frac{d u}{d r}
$$

for laminar flow in which $\tau_{l}$ is the laminar shearing stress and $\mu$ denotes the viscosity of the fluid. On this basis the Navier-Stokes equation for turbulent flow can be written in cylindrical coordinates form as:

$$
\frac{1}{r} \frac{d}{d r}\left[\left(\mu+A_{\tau}\right) r \frac{d u}{d r}\right]+h=0
$$

where $h=d p / d x$ is the presure gradient along the pipe.

\section{Prandtl's mixing length hypotesis.}

If $l$ is the mixing length then:

$$
u^{\prime} \sim l \frac{d u}{d r}
$$

The shearing stress can be expressed as:

$$
\tau=\rho l^{2}\left(\frac{d u}{d r}\right)^{2}
$$

and the apparent, or eddy viscosity as:

$$
A_{\tau}=\rho l^{2}\left(\frac{d u}{d r}\right)
$$

where $\rho$ denotes the density of the fluid.

\section{Kinetic energy of pulsation $E^{\prime}$}

The kinetic energy of turbulent pulsation may be expressed as a function of the mixing length. If $\mathrm{E}^{\prime}$ is the kinetic energy then:

$$
\mathrm{E}^{\prime} \sim \rho u^{\prime 2}
$$

and using the expression for $u^{\prime}$ given by Prandtl's hypotesis it follows that:

and:

$$
\mathrm{E}^{\prime} \sim \rho l^{2}\left(\frac{d u}{d r}\right)^{2}
$$

$$
\mathrm{E}^{\prime}=c \rho l^{2}\left(\frac{d u}{d r}\right)^{2}
$$

in which $c$ is some constant of proportionality.

Balance of pulsation energy.

Since the flow is fully developed it will have an established turbulence $\left(\check{A}_{\tau}\right.$ and $\mathrm{E}^{\prime}$ are independent of time). Therefore the losses in kinetic energy of turbulent motion which are caused by the viscous effects must be compensated continously to maintain a constant degree of turbulence at a given point. The feeding of turbulence (for instance from the wall) will be produced by a turbulent exchange in which the mixing length plays an important role.

The "balance of pulsation energy" equation may be wrilten as follows:

The flux of turbulent energy entering an arbitrary element with a closed surface $S$ must be equal to the rate of change of losses of kinetic energy in the turbulent pulsation due to the viscous effects in the volume $V$ enclosed by the surface $S$.

Consider an element with normal $\vec{n}$. Transport of turbulent energy in the $\vec{n}$ direction through an element is assumed to be produced if the mixing lengths on both sides of the element are different, i.e., the flux of turbulent energy depends on:

$$
\frac{d l}{d n} \quad \text { or }(\operatorname{grad} l, \vec{n})
$$

The flux of energy through a closed surface $S$ will be:

$$
\frac{1}{a} \iint_{s}(\operatorname{grad} l, \vec{n}) d \mathrm{~S}
$$

where $\alpha$ is a coefficient depending on overall characteristics of the flow, i.e., on the intensity of the turbulence.

This energy flux compensates for the loss rate in kinetic energy of the turbulent pulsation in the volume $V$ surrounded by the surface $S$. The kinetic energy is proportional to $\mathrm{E}^{\prime}$, being the rate of loss:

$$
\iiint_{v} \mathrm{E}^{\prime} d \mathrm{~V}
$$

The equation of balance is then:

$$
\iint_{S}(\operatorname{grad} l, \vec{n}) d \mathbf{S}+a \iiint_{V} \mathrm{E}^{\prime} d \mathrm{~V}=0
$$


By the divergence theorem:

$$
\iiint_{\mathrm{V}}\left(\operatorname{div} \operatorname{grad} l+a \mathrm{E}^{\prime}\right) d \mathrm{~V}=0
$$

and since the volume is arbitrary:

Or:

$$
\text { div grad } l+a \mathrm{E}^{\prime}=0
$$

$$
\nabla^{2} l+a \mathrm{E}^{\prime}=0
$$

which in cylindrical coordinates is expressed as:

$$
\frac{1}{r} \frac{d}{d r}\left(r \frac{d l}{d r}\right)+a \mathrm{E}^{\prime}=0
$$

\section{Complete system of equations for turbulent flow in a circular} pipe.

From the above it is clear that the complete system of equations for turbulent flow in a circular pipe is given by:

$$
\begin{gathered}
\frac{d u}{d r}+\frac{u}{r}=0 \\
\frac{1}{r} \frac{d}{d r}\left[\left(\mu+\mathrm{A}_{\tau}\right) r \frac{d u}{d r}\right]+h=0 \\
\mathrm{~A}_{\tau}=\rho l^{2}\left(\frac{d u}{d r}\right) \\
\mathrm{E}^{\prime}=c \rho l^{2}\left(\frac{d u}{d r}\right)^{2} \\
\frac{1}{r} \frac{d}{d r}\left(r \frac{d l}{d r}\right)+a \mathrm{E}^{\prime}=0
\end{gathered}
$$

\section{Solution of the system of equations.}

Integrating equation (2) leads to:

$$
\left(\mu+A_{\tau}\right) r \frac{d u}{d r}+\frac{h r^{2}}{2}=\mathrm{C}_{1}
$$

where $C_{1}$ is the constant of integration.

Since when:

$$
r=0, \quad \frac{d u}{d r}=0, \quad \mathrm{C}_{1}=0
$$

and :

$$
\left(\mu+\mathrm{A}_{\tau}\right) \frac{d u}{d r}=-\frac{h r}{2}
$$

For a high degree of turbulence the eddy viscosity is much larger than the laminar viscosity and therefore $\mu$ can be neglected, except in the region close to the wall where the viscous effects are predominant. The above equation then becomes:

$$
A_{\tau} \frac{d u}{d r}=-\frac{h r}{2}
$$

Introducing $\mathrm{E}^{\prime}$ and $\mathrm{A}_{\tau}$ in equations (3) and (6) and considering that $(d u / d r)<0$, yields:

$$
\rho l^{2}\left(\frac{d u}{d r}\right)^{2}=\frac{h r}{2}
$$

$$
\frac{1}{r} \frac{d}{d r}\left(r \frac{d l}{d r}\right)=-a c \rho l^{2}\left(\frac{d u}{d r}\right)^{2}
$$

Replacing:

$$
\rho l^{2}\left(\frac{d u}{d r}\right)^{2} \quad \text { with }: \frac{h r}{2}
$$

reduces equation (8) to:

$$
\frac{1}{r} \frac{d}{d r}\left(r \frac{d l}{d r}\right)=-\frac{a c}{2} h r
$$

After integration:

$$
r \frac{d l}{d r}=-\frac{a c}{6} h r^{3}+\mathrm{C}_{2.2}
$$

where $C_{2}$ is the constant of integration.

Since when:

$$
\begin{gathered}
r=0, \quad \frac{d l}{d r}=0 \quad \text { and hence: } \quad \mathrm{C}_{22}=0 \\
\frac{d l}{d r}=-\frac{a c}{6} h r^{2}
\end{gathered}
$$

After integrating again:

$$
l=--\frac{a c}{18} h r^{3}+\mathrm{C}_{3}
$$

in which $\mathrm{C}_{3}$ denotes the constant of integration. Since on the wall there is no pulsation, at $r=r_{0}$, $l=0$.

Thus:

$$
\mathrm{C}_{3}=\frac{a c}{18} h r_{0}{ }^{3}
$$

and:

$$
l=\frac{a c}{18} h r_{0}^{3}\left[1-\left(\frac{r}{r_{0}}\right)^{3}\right]
$$

If the mixing length on the center of the pipe is:

then:

$$
l_{10}=\frac{a c}{18} h r_{0}^{3}
$$

$$
l==l_{0}\left[1-\left(\frac{r}{r_{0}}\right)^{3}\right]
$$

Introducing this expression in equation (7) gives:

$$
\rho l^{2}\left(\frac{d u}{d r}\right)^{2}=\frac{h r}{2}
$$

and considering that $(d u / d r)<0$ :

$$
\frac{d u}{d r}=-\frac{1}{l_{0}} \sqrt{\frac{h r_{0}}{2 \rho}} \frac{\sqrt{\left(r / r_{0}\right)}}{\left[l-\left(r / r_{0}\right)^{3}\right]}
$$

Introducing now the concept of the shear velocity:

$$
v_{*}=\sqrt{\frac{h r_{0}}{2 \rho}}
$$

and the dimensionless coordinate $r / r_{0}$ produces:

$$
\frac{d\left(u / v_{*}\right)}{d\left(r / r_{0}\right)}=-\frac{r_{0}}{l_{0}} \frac{V\left(r / r_{0}\right)}{1-\left(r / r_{0}\right)^{3}}
$$


Calling $\xi=\left(r / r_{0}\right)$, and noting that:

$$
\int \frac{\sqrt{\xi}}{1-\xi^{3}} d \xi=\frac{2}{3} \tanh ^{-1} \xi^{3 / 2}
$$

leads to the difference between the value of the velocity at the axis of the pipe $U$ and the velocity at a given point $u$ in the form:

$$
\frac{\mathrm{U}-u}{\nu_{*}}=\frac{2}{3} \frac{r_{0}}{l_{0}} \tanh ^{-1}\left(\frac{r}{r_{0}}\right)^{3 / 2}
$$

Von Karman introduced the "universal constant" $x$, which characterizes the rate of growth of the mixing length at the wall:

$$
x=\left.\frac{d l}{d r}\right|_{r=r_{i}}
$$

Differentiating equation (10) gives at $r=r_{0}$ :

$$
x=3 \frac{l_{0}}{r_{0}}
$$

Thus:

$$
\frac{r_{0}}{l_{0}}=\frac{3}{x}
$$

and:

$$
\frac{\mathrm{U}-u}{v_{*}}=\frac{2}{x} \tanh ^{-1}\left(\frac{r}{r_{0}}\right)^{3 / 2}
$$

From the available experimental data the accepted value of $x$ is 0.40 ; hence:

$$
\frac{\mathrm{U}-u}{v_{*}}=5.0 \tanh ^{-1}\left(\frac{r}{r_{0}}\right)^{3 / 2}
$$

This is an expression for the universal law of the velocity distribution in a circular pipe.

From equation (11):

$$
l_{0}=x \frac{r_{0}}{3}
$$

and making the substitution in equation (10):

$$
l=\frac{x}{3} r_{0}\left[1-\left(\frac{r}{r_{0}}\right)^{3}\right]
$$

which is the expression for the mixing length.

From the expression:

$$
\mathrm{A}_{\tau}=\rho l^{2}\left|\frac{d u}{d r}\right|
$$

and the obtained results for $l$ and:

$$
\frac{d u}{d r}=-\frac{3 v_{*}}{\chi r_{0}} \frac{\sqrt{\left(r / r_{0}\right)}}{l-\left(r / r_{0}\right)^{3}}
$$

the expression for the eddy viscosity is:

$$
\mathrm{A}_{\tau}=\frac{x}{3} \rho r v_{*} \sqrt{\frac{r}{r_{0}}}\left[1-\left(\frac{r}{r_{0}}\right)^{3}\right]
$$

After some rearrangement the above result is expressed in terms of the kinematic eddy viscosity:

$$
\frac{\varepsilon}{v_{*} r}=\frac{x}{3} \sqrt{\frac{r}{r_{0}}}\left[1-\left(\frac{r}{r_{0}}\right)^{3}\right]
$$

where $\varepsilon$ denotes the kinematic eddy viscosity.

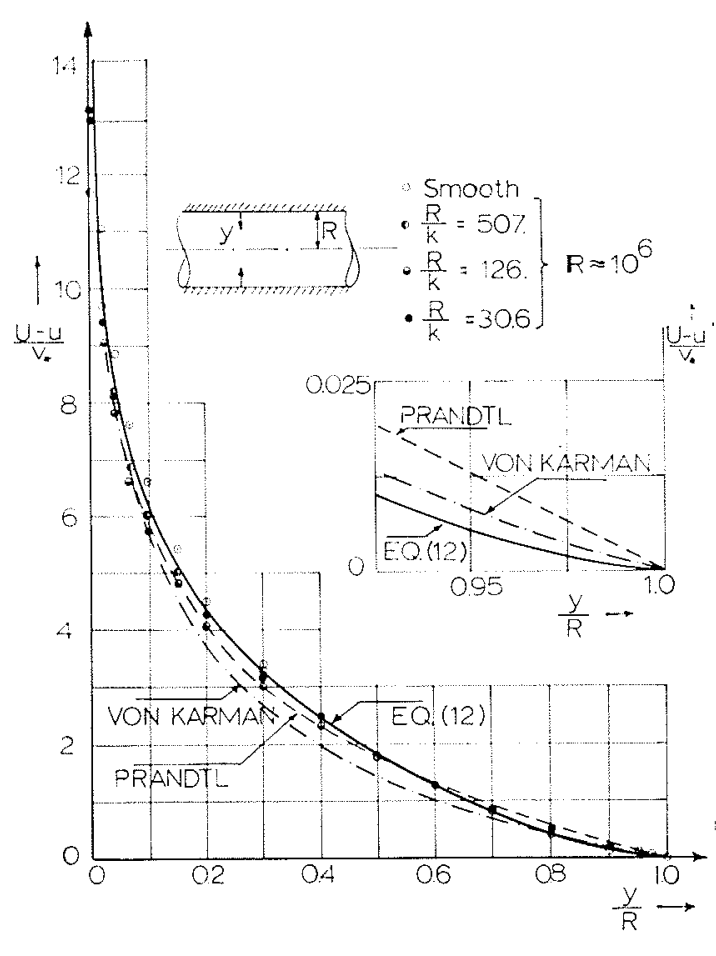

1/ Velocity distribution.

Répartition des vitesses.

2/ Mixing length distribution.

Répartition des longueurs de mélange.

3/ Kinematic eddy viscosity distribution.

Répartition des viscosités cinématiques tourbillonnaires.

\section{Analysis of results}

The equation obtained for the velocity distribution :

$$
\frac{\mathrm{U}-u}{v_{*}}=\frac{2}{x} \tanh ^{-1}\left(\frac{r}{r_{0}}\right)^{: / 2}
$$

covers the region from the axis of the pipe to $\Gamma_{0} \pm \delta$, where $\delta$ is the thickness of the laminar sublayer. This expression is compared with experimental data obtained by Nikuradse (*) and other expressions in Figure 1.

To compare this new result with the already known ones it is convenient to consider approximate expressions for $\tanh ^{-1}$ in the case of small and large values of $r / r_{0}$.

In the region close to the wall, where $r / r_{0}$ is large, and denoting:

$$
\begin{gathered}
y=r_{0}-r \\
\left(\frac{r}{r_{0}}\right)^{3 / 2}=\left(1-\frac{y}{r_{0}}\right)^{3 / 2} \approx 1-\frac{3}{2} \frac{y}{r_{0}}+\ldots
\end{gathered}
$$

(*) H. Schlichting. - Boundary layer theory. Pergamon Press, 1955, p. 409. 


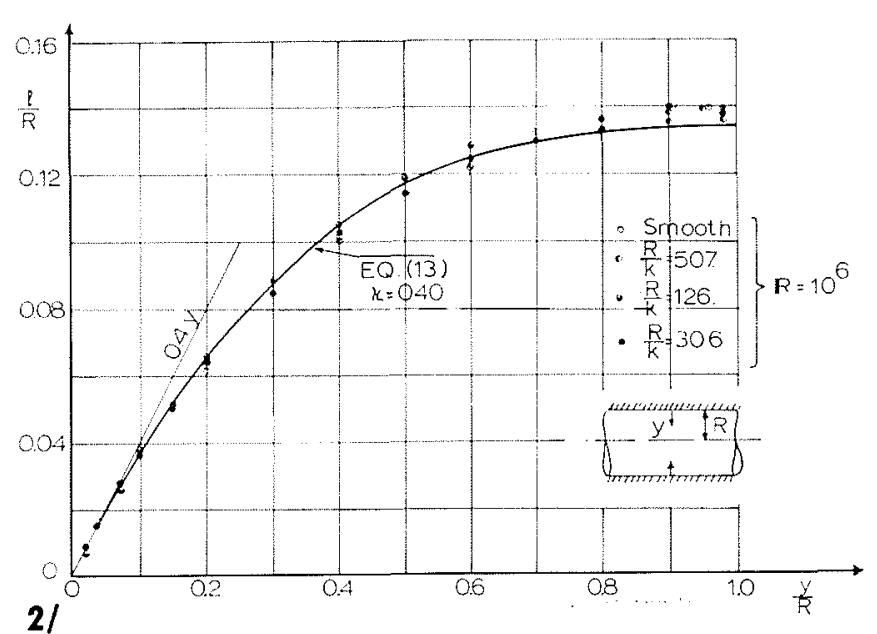

2/

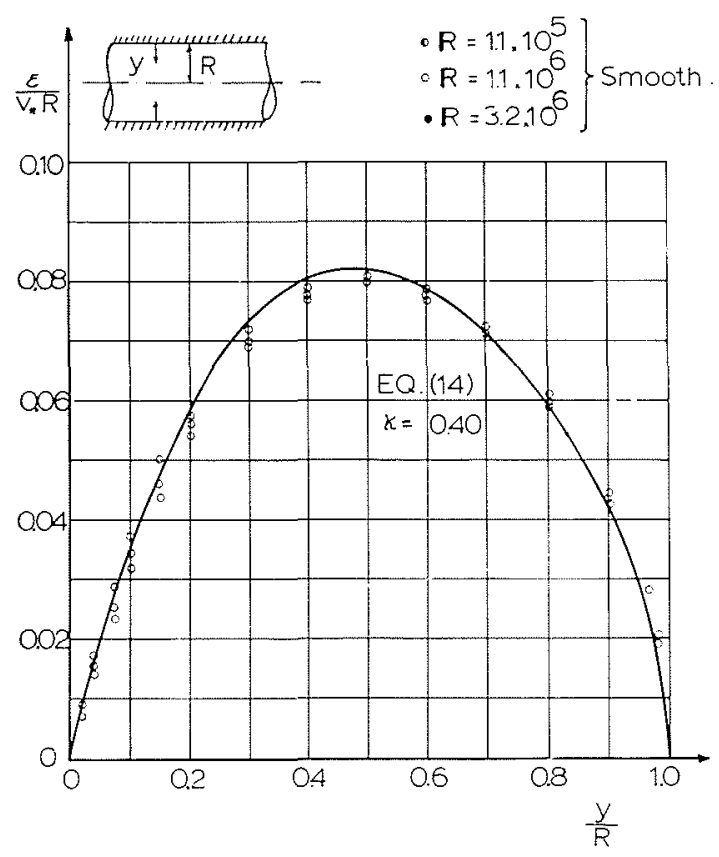

Thus :

$$
\begin{aligned}
\tanh ^{-1}\left(\frac{r}{r_{0}}\right)^{3 / 2} & =\frac{1}{2} \ln \frac{1+\left(r / r_{0}\right)^{3 / 2}}{l-\left(r / r_{0}\right)^{3 / 2}} \\
& =\frac{1}{2} \ln \frac{r_{0}}{y}+\frac{1}{2} \ln \frac{4}{3}+\ldots
\end{aligned}
$$

and it follows that:

$$
\frac{\mathrm{U}-u}{v_{*}}=\frac{1}{x} \ln \frac{r_{0}}{y}+\frac{1}{x} \ln \frac{4}{3}
$$

For small values of $y / r_{0}$ this formula practically coincides with the expression:

$$
\frac{\mathrm{U}-u}{D_{*}}=\frac{1}{x} \ln \frac{r_{0}}{y}
$$

which is the well know expression given by Prandtl.

For the central zone where $r / r_{0}$ is small:

$$
\tanh ^{-1}\left(\frac{r}{r_{0}}\right)^{3 / 2}=\left(\frac{r}{r_{0}}\right)^{3 / 2}+\ldots
$$

Therefore at points close to the axis for $x=0.40$ :

$$
\frac{\mathrm{U}-u}{v_{*}}=5.0\left(\frac{r}{r_{0}}\right)^{3 / 2}
$$

which has a zero tangent when $r \rightarrow 0$. This expression is remarkable close to the empirical formula given by Darcy in 1855 :

$$
\frac{\mathrm{U}-u}{v_{*}}=5.08\left(\frac{r}{r_{0}}\right)^{3 / 2}
$$

The equivalence of the functional relations and even the numerical values of the coefficients confirms the validity of the theory and the numerical value of the Karman universal constant.

The expression for the mixing length is given as:

$$
l=\frac{x}{3} r_{0}\left[1-\left(\frac{r}{r_{0}}\right)^{3}\right]
$$

This expression can be analyzed for the case where $y / r_{0}$ is small (region close to the wall):

$$
\left(\frac{r}{r_{0}}\right)^{3}=\left(1-\frac{y}{r_{0}}\right)^{3}=1-3 \frac{y}{r_{0}}+\ldots
$$

Thus:

$$
l=x y
$$

which is the Prandtl's expression.

For the central region the analytical results obtained for the mixing length are quite far from Prandtl's approximation and are remarkably close to the experimental points obtained by Nikuradse, (see Fig. 2).

Similarly the analytical results for the distribution of eddy viscosity agrees with experimental points throughout the whole pipe (see Fig. 3 ).

\section{Conclusions}

The results obtained in the present paper show that the basic equation for turbulent flow developed by $\mathrm{A}$. Zagustin allow an analytical solution for the problem of a turbulent flow in a smooth pipe. These solutions are obtained without making any empirical or semi-empirical assumptions aboul the mixing lenght distribution, as it is done in many papers. The agreement between theory and experiments is excellent not only at the neighbourhood of the wall but also throughout the whole pipe. It should be pointed out that since all the final expressions depend on only one experimental constant, namely the Karman's universal constant $x$, its numerical value will determine the behaviour of the theoretical curves. In the graphs presented here the value of $x$ was taken as 0.40 , which is the standard value accepted in the literature. However if a value slightly smaller than 0.40 is taken, for instance $x=0.395$ or 0.39 , then the agreement between the theoretical results and experiments is even better. It is of a particular interest the fact that the expression for the velocity distribution coincides at the centerline with the widely used old empirical formula developed by Darcy in 1855, in which there is a zero tangent at the axis, and with 
Prandtl's expression at the neighbourhood of the wall, where the mixing length varies linearly with the distance. Both cases are "clamp" by the obtained theoretical solution so that the agreement with experiments in the middle region is also good.

\section{Appendix - Notation}

The following symbols have been adopted for use in this paper:

a constant of proportionality;

$A_{\tau}$ eddy viscosity;

$c$ constant of proportionality;

$\mathrm{E}^{\prime} \quad$ kinetic energy of pulsation;

$h$ pressure gradient along the pipe;

$l$ Prandtl's mixing length;

$\vec{n} \quad$ normal to any given surface;

$p$ pressure;

$r$ radial distance measured from the axis of the pipe; $r_{0}$ radius of the pipe;

$\vec{r} \quad$ position vector;

$S$ closed surface;

$t$ lime;

$\mathrm{T}$ interval of time;

"l velocity at a given point in the pipe;

$u^{\prime}$ pulsation velocity in the $r$ direction;

$\mathrm{U}$ velocity at the axis of the pipe;

$n_{*}$ shear velocity;

$\mathrm{V}$ volume of the element surrounded by the sur-

$\rightarrow \quad$ face $\mathrm{S}$;

$\vec{V} \quad$ velocity veclor;

$\overrightarrow{\vec{V}}$ time-average velocity;

$\vec{V}^{\prime}$ pulsation velocity;

$y$ distance measured from the wall;

$x$ Karman' universal constant;

$\nabla^{2}$ div grad;

$\tau_{l} \quad$ laminar shear stress;

$\tau_{t} \quad$ turbulent shear slress;

$\mu$ laminar fluid viscosity;

$\nu$ kinematic fluid viscosity;

$\varepsilon \quad$ kinematic eddy viscosity.

$\varphi$ fluid density.

\title{
Résumé
}

\section{Solution analytique pour les régimes turbulents dans les tuyaux}

\author{
par A. Zagustin * et K. Zagustin **
}

On examine un écoulement turbulent en conduite bien établi, et on complète à l'aide d'une équation de base de l'écoulement turbulent l'ensemble d'équations différentielles nécessaire à la résolution du problème par voie analytique. On obtient de nouvelles relations simples tenant compte de la répartition des vitesses, de la longueur de mélange, et de la viscosité tourbillonnaire, et on les confronte avec les formules et les données expérimentales existantes. La simplicité et l'excellent comportement des courbes théoriques, ainsi que la bonne concordance de ces relations avec les données expérimentales, démontrent la validité du présent procédé théorique de résolution du problème. 